\title{
Quantum Chemical Approach for Condensed-Phase Thermochemistry(IV): Solubility of Gaseous Molecules
}

\author{
Atsushi Ishikawa ${ }^{\mathrm{a}}$, Masahiro Kamata ${ }^{\mathrm{b}}$, and Hiromi Nakai ${ }^{\mathrm{a}-\mathrm{d}, ~ *}$ \\ ${ }^{a}$ Research Institute for Science and Engineering, Waseda University, 3-4-1 Okubo, Shinjuku, Tokyo 169-8555, Japan \\ ${ }^{\mathrm{b}}$ Department of Chemistry and Biochemistry, School of Advanced Science and Engineering, Waseda University, 3-4-1 \\ Okubo, Shinjuku, Tokyo 169-8555, Japan \\ ${ }^{c}$ CREST, Japan Science and Technology Agency, 4-1-8 Honcho, Kawaguchi, Saitama 332-0012, Japan \\ ${ }^{\mathrm{d}}$ ESICB, Kyoto University, Kyotodaigaku-Katsura, Kyoto 615-8520, Japan \\ ${ }^{*}$ To whom correspondence should be addressed. E-mail: nakai@waseda.jp
}

(C) 2016. This manuscript version is made available under the Elsevier user license

http://www.elsevier.com/open-access/userlicense/1.0/ 


\begin{abstract}
The harmonic solvation model (HSM) was applied to the solvation of gaseous molecules and compared to a procedure based on the idealgas model (IGM). Examination of 25 moleculesshowed that (i) the accuracy of $\Delta G_{\text {solv was }}$ similar forboth methods, but the HSM shows advantages for calculating $\Delta H_{\text {solv }}$ and $T \Delta S_{\text {solv }}$;(ii) $\Delta S_{\text {solv }}$ contributes morethan $\Delta H_{\text {solv }}$ to $\Delta G_{\text {solv }}$ in the HSM, i.e. the solvation of gaseous molecules is entropy-driven,which agrees well with experimental understanding(the IGM does not show this); (iii) the temperature dependence of Henry's law coefficientwas correctly reproduced with the HSM.
\end{abstract}

Keywords: condensed-phase; quantum chemistry; gaseous solubility; harmonic solvation model; idealgas model 


\section{Introduction}

The solubility of gaseousmoleculesis an important parameter in vapor-liquid equilibriumsin basic physical chemistry andenvironmental science. Solubility is often expressed by the more clearly defined Henry's law coefficient (HLC). Accurate knowledge of solubility is essential for predicting the environmental behavior of pollutants, since it describes the ability to distribute between vapor- and liquid-phases[1]. Extensiveefforthas been devoted to accurate estimation of the HLC.However, limited experimental solubility data have beenreported, partly owing to difficulties in obtaining accurate data by experimental techniques,such as adsorption effects on the walls of apparatus and analytical limits of detection[2,3].Altschuh and Brüggemannrevealed in 1993 that solubility data has been reported for only $17.4 \%$ of approximately 800 environmentally relevant chemicals[3].

The difficulties associated with experimental techniques increase the demand for theoretical and computational methods. In chemical engineering, computer simulations based on property-property relationships (PPRs) or structure-property relationships (SPRs) have been widely used. Quantitative structure-property relationships (QSPRs) are considered accurate models for predictinggaseous solubility or the HLC[4-7].This method has been successfully applied to various chemical compounds, although the accuracyof the fitting parameters strongly depends on the quality of the experimental data, such as vapor pressure or aqueous solubility, and such dataare often unavailable or unreliable.

Theoretical methods, such as molecular dynamics (MD) andMonte-Carlo (MC) simulations, are less dependent on the quality of experimental data[8-10].Typical approaches are the grand canonical MC with histogram reweighting method,Gibbs-Duhem integration method,isothermal-isobaric $(N P T)$ ensemble 
simulationwith the Widom test particle insertion method,etc.[11-13]. These simulation techniques give accurate

HLCs, and can predictnot only the HLC at a fixed temperature, but also itstemperature dependence[9,10,14].However, their accuracy issignificantly dependent on the simulation conditions, such as selection of the molecular model (all-atom or united-atom),force field, number of molecules in the simulation box, etc.[15-17].Particular attention should therefore be paid to the selection of simulation conditions, since the vapor-liquid equilibrium is an intrinsically multi-component system. For example, the Lennard-Jones parameter for solute-solvent pairs should be carefully selected. Several studies have shown that simple formulae, such as the Lorentz-Berthelot rule for estimating Lennard-Jones parameters for different compound pairs,are unsatisfactory for the reliable prediction of gaseous solubility. Several approaches have been proposed to improve the Lorentz-Berthelot rule, however, many require additional information such as polarizability data[18,19].Force field selection is a difficult problem in vapor-liquid simulations. State-of-the-art force fields, such as polarizable force fields or fluctuation charge, significantly improve the simulation results for liquid systems. However, as reported by Panagiotopoulos, the polarizable force field does notalways improve the thermodynamic properties for vapor-liquid equilibriums, such as vapor pressure or heat of vaporization[20].Recent techniques, such asthe reaction Gibbs ensemble MC (RGEMC),show promise for circumventing the above-stated force field problem[21,22].

Another theoretical tool used for calculating solubility is the ab initioor quantum chemistry approach[23,24],whichhas been applied to the estimation of HLCs[25-27].However, the current quantum chemistry approach still presents problems in its basic part and needs improvement.Zhang and Zenghave shown that direct 
evaluation of the HLC from the Gibbs energy of solvation $\left(\Delta G_{\text {solv }}\right)$ is not sufficiently accurate, and proposed the use of a reference substance and the relative Gibbs energy [28,29].Another disadvantage of the quantum chemistrymethod is that the temperature dependence of the HLC is rarely provided. In previous studies employing the quantum chemistry method, HLCs were only evaluated at a fixed temperature and their temperature dependence was not discussed[25-27].It is widely recognized that the temperature dependence of the vapor-liquid equilibriumcan be described with the help of statistical thermodynamics, as shown by Klamt et al.in the Conductor-like Screening Model for Real Solvents (COSMO-RS)approach [30,31].Temperature-dependent aqueous solubilities were investigated with the COSMO-RS method showing good agreement with experimental data [31-33].

One of the most serious problems associated withthe quantum chemistry method when applied to vapor-liquid phase co-existing systems is its insufficient description of the liquid-phase entropy. Recently, we proposed a novel quantum chemical modelaimed at accurate thermodynamic property calculation in the condensed-phase[34]. The method is called the harmonic solvation model (HSM), since the solute's motion in the solvent environment is expressed by the harmonic oscillator.The calculation of thermodynamic properties, such as enthalpy, entropy, and Gibbs energy, are improved with the HSM, especially the entropy term.

In the present study, we used the HSM to calculate the HLC of simple gaseous molecules.One advantage of the HSM over conventional quantum chemistry methods is that $\Delta H_{\text {solv }}$ and $\Delta S_{\text {solv }}$ can be accurately evaluated as well as $\Delta G_{\text {solv. }}$ Previous studies applyingquantum chemistry methods to the solubility problem mainly focus on $\Delta G_{\text {solv }}$ without detailed discussion of individual enthalpy and entropy contributions, although $\Delta H_{\text {solv }}$ and $\Delta S_{\text {solv }}$ 
clearly have different physical and chemical origins[35]. Such discussion would be advantageous in calculating the temperature dependence of the HLC, since it is mainly determined by $\Delta S_{\text {solv }}$. 


\section{Theoretical Aspects}

\section{A. HSM}

In the quantum chemistry method, vapor-phase thermodynamic properties are calculated from the set of equations formulated by the kinetic theory of gas underthe idealgas assumption; the ideal gas model (IGM). In the IGM, the motion of gaseous molecules is separated into vibrational, rotational, and translational components, whose energies ( $E_{\mathrm{vib}}$ and $E_{\text {rot }}$, respectively) and entropies $\left(S_{\mathrm{vib}}, S_{\text {rot }}\right.$, and $S_{\text {trans }}$, respectively) are expressed by Eqs.

$$
\begin{aligned}
& E_{\text {vib }}=N_{\mathrm{A}} k_{\mathrm{B}} T \sum_{i}^{3 N-6} \frac{h v_{i}}{k_{\mathrm{B}} T}\left\{\frac{1}{2}+\frac{\exp \left(-h v_{i} / k_{\mathrm{B}} T\right)}{1-\exp \left(-h v_{i} / k_{\mathrm{B}} T\right)}\right\}, \\
& E_{\text {rot }}^{\mathrm{IGM}}=E_{\text {trans }}^{\mathrm{IGM}}=\frac{3}{2} N_{\mathrm{A}} k_{\mathrm{B}} T=\frac{3}{2} R T, \\
& S_{\text {vib }}=N_{\mathrm{A}} k_{\mathrm{B}} \sum_{i}^{3 N-6}\left[\frac{\left(h v_{i} / k_{\mathrm{B}} T\right) \exp \left(-h v_{i} / k_{\mathrm{B}} T\right)}{1-\exp \left(-h v_{i} / k_{\mathrm{B}} T\right)}-\ln \left\{1-\exp \left(-h v_{i} / k_{\mathrm{B}} T\right)\right\}\right], \\
& S_{\text {rot }}^{\mathrm{IGM}}=R\left\{\frac{1}{2} \ln A B C+\frac{3}{2} \ln T-\ln \sigma+\text { const. }\right\}, \\
& S_{\text {trans }}^{\mathrm{IGM}}=R\left\{\frac{3}{2} \ln M+\frac{5}{2} \ln T-\ln p+\text { const. }\right\} .
\end{aligned}
$$

$N_{\mathrm{A}}, k_{\mathrm{B}}, h$, and $R$ are the Avogadro, Boltzmann, Planck, and gas constants, respectively. $p$ and $T$ are pressure and temperature, and the vibrational frequencies $\left\{v_{i}\right\}$, mass $M$, rotational constants $\{A, B$, and $C\}$, and symmetry number $\sigma$ are system-dependent variables. The enthalpy and Gibbs energy are given by

$$
H=E_{\mathrm{elec}}+E_{\mathrm{vib}}+E_{\mathrm{rot}}+E_{\mathrm{trans}}+W
$$

and

$$
G=H-T S=H-T\left(S_{\text {elec }}+S_{\text {vib }}+S_{\text {rot }}+S_{\text {trans }}\right),
$$


respectively, where work $W=p V$. Note that $S_{\text {elec }}$ is zero in the ground electronic state for closed-shell molecules. In the condensed-phase, rotational and translational motionsarerestricted by solute-solvent interactions. Consequently, expressions for rotational and translational energies and entropies based on the IGM (Eqs.(2), (4), and(5)) are inappropriate in the condensed-phase.It should be noted that many quantum chemistry programs employ the IGM formulae for the calculation of condensed-phase thermodynamic properties.

To adequately express these effects, the HSM treats the rotational and translational motions corresponding to solute-solvent interactions as harmonic oscillators[34]. By applying the harmonic oscillator model to the translational and rotational motions, their energy and entropy contributions become

$$
E_{\text {trans/rot }}^{\mathrm{HSM}}=\frac{1}{2} N_{\mathrm{A}} k_{\mathrm{B}} T \sum_{i \in \text { trans/rot }}^{3} \frac{h v_{i}}{k_{\mathrm{B}} T}\left\{\frac{1}{2}+\frac{\exp \left(-h v_{i} / k_{\mathrm{B}} T\right)}{1-\exp \left(-h v_{i} / k_{\mathrm{B}} T\right)}\right\}
$$

and

$$
S_{\text {trans/rot }}^{\mathrm{HSM}}=N_{\mathrm{A}} k_{\mathrm{B}} \sum_{i \in \text { trans/rot }}^{3}\left[\frac{\left(h v_{i} / k_{\mathrm{B}} T\right) \exp \left(-h v_{i} / k_{\mathrm{B}} T\right)}{1-\exp \left(-h v_{i} / k_{\mathrm{B}} T\right)}-\ln \left\{1-\exp \left(-h v_{i} / k_{\mathrm{B}} T\right)\right\}\right] \text {, }
$$

respectively. Note that $S_{\text {trans/rot }}^{\mathrm{HSM}}$ is identical to $S_{\text {vib }}^{\mathrm{IGM}}$ (Eq.(3)). Therefore, a key difference between the IGM and HSM is the treatment of the solute rotational and translational motions in the condensed-phase; in the IGM, the rotational and translational modes, which normally correspond to the six lowest modes, are omitted when evaluating the thermal contribution from vibrational motion. In contrast, all motions of the solute molecules are treated as harmonic vibrations in the HSM. Therefore, the rotational and translational energies and entropies were evaluated according to the vibrational formulae. 
The vibrational frequency in the condensed-phase is obtained using the framework of the self-consistent reaction field (SCRF) procedure. In the HSM, the Hessian matrix is evaluated by fixing solute cavities since interactions between a solute molecule and its cavities are presumed to be solute-solvent interactions. After the Hessian matrix evaluation, vibrational, rotational, and translational energies and entropies are evaluated.

In the present study, $\Delta G_{\text {solv }}$ was calculated using the following formula in both theIGM and HSM;

$$
\begin{aligned}
\Delta G_{\mathrm{solv}} & =\left[E_{\mathrm{elec}}^{\mathrm{sol}}+E_{\mathrm{vib}}^{\mathrm{sol}}+E_{\mathrm{rot}}^{\mathrm{sol}}+E_{\mathrm{trans}}^{\mathrm{sol}}+p V-T\left(S_{\mathrm{elec}}^{\mathrm{sol}}+S_{\mathrm{vib}}^{\mathrm{sol}}+S_{\mathrm{rot}}^{\mathrm{sol}}+S_{\mathrm{trans}}^{\mathrm{sol}}\right)\right] \\
& -\left[E_{\mathrm{elec}}^{\mathrm{gas}}+E_{\mathrm{vib}}^{\mathrm{gas}}+E_{\mathrm{rot}}^{\mathrm{gas}}+E_{\mathrm{trans}}^{\mathrm{gas}}+p V-T\left(S_{\mathrm{elec}}^{\mathrm{gas}}+S_{\mathrm{vib}}^{\mathrm{gas}}+S_{\mathrm{rot}}^{\mathrm{gas}}+S_{\mathrm{trans}}^{\mathrm{gas}}\right)\right]=\Delta H_{\mathrm{solv}}-T \Delta S_{\mathrm{solv}}
\end{aligned}
$$

where both vapor- and liquid-phase Gibbs energies include all components. 


\section{B. Solubility and HLC}

The vapor- and liquid-phase chemical potentials are written as

$$
\mu_{i, \text { vap }}(p, T)=\mu_{i, \text { vap }}^{\circ}(p, T)+R T \ln \left(p_{i} / p_{0}\right)
$$

and

$$
\mu_{i, \text { liq }}\left(p, T, x_{i}\right)=\mu_{i \text {.liq }}^{\circ}(p, T)+R T \ln a_{i}=\mu_{i \text {.liq }}^{\circ}+R T \ln \gamma_{i} x_{i},
$$

respectively, where $p_{i}$ and $p_{0}$ are the partial pressure of compound $i$ and the reference pressure, respectively; $a_{i}$ is the

activity of $i$; and $\gamma_{i}$ and $x_{i}$ are the activity coefficient and solubility (in molar fraction) of $i$, respectively[14,36,37].Since these chemical potentials are equal at vapor-liquid equilibrium,

$$
\mu_{i, \text { vap }}^{\circ}+R T \ln \left(p_{i} / p_{0}\right)=\mu_{i, \text { liq }}^{\circ}+R T \ln \gamma_{i} x_{i}
$$

$p_{i}$ and $x_{i}$ are therefore related by

$$
p_{i}=K_{i} x_{i}
$$

in which

$$
K_{i}(p, T)=p_{0} \gamma_{i} \exp \left[\frac{\mu_{i, \text { liq }}^{\circ}(p, T)-\mu_{i, \text { vap }}^{\circ}\left(p_{0}, T\right)}{R T}\right]
$$

The HLC $H_{i}(p, T)$ is defined as the limiting value of $K_{i}(p, T)$ as[38]

$$
H_{i}(p, T)=\lim _{x_{i} \rightarrow 0}\left(\frac{p_{i}}{x_{i}}\right)=\lim _{x_{i} \rightarrow 0} K_{i}(p, T) .
$$

$H_{i}(p, T)$ depends on both pressure and temperature, but its pressure dependence is negligible. Therefore, we consider only its temperature dependence. 
In MD and MC simulations, the HLC is calculated via the excess chemical potential (at infinite dilution) $\mu_{\mathrm{ex}}^{\infty}$. This corresponds to the non-ideality of the solution, i.e. solute-solvent interactions. Since $\gamma_{i}$ is related to $\mu_{\mathrm{ex}}^{\infty}$ as $\gamma_{i}=\exp \left(\mu_{\mathrm{ex}}^{\infty} / R T\right)$, the HLC is calculated as

$$
H_{i}(T)=p_{0} \exp \left(\frac{\mu_{\mathrm{ex}}}{R T}\right)=\rho_{i} R T \exp \left(\frac{\mu_{\mathrm{ex}}^{\infty}}{R T}\right)
$$

where $\rho_{i}$ is the density of $i$ in the liquid-phase. In the quantum chemistrymethod, the ideal and non-ideal parts of $\Delta G_{\text {solv }}$ are treated together as the whole solvation free energy $\Delta \mu_{\text {solv }}$. The HLC is calculated as

$$
H_{i}(T)=p_{0} \exp \left(\frac{\mu_{i, l}^{\circ}-\mu_{i, g}^{\circ}+\mu_{\mathrm{ex}}^{\infty}}{R T}\right)=p_{0} \exp \left(\frac{\Delta \mu_{\mathrm{solv}}}{R T}\right)
$$

In many cases,the reference state is taken as the standard condition for solution $(1 \mathrm{~mol} / \mathrm{L})$ and in this case $p_{0}$ becomes $R T$. This definition was also applied in the present study, i.e. the solvation chemical potential (or Gibbs energy) is defined as the Gibbs energy of transferring $1 \mathrm{~mol} / \mathrm{L}$ vapor-phase molecules into solution[39]. Since many experimental and theoretical studies report the HLC as

$$
\ln H_{i}(T)=\frac{\Delta \mu}{R T}+\ln R T
$$

wehave done the same herein. 


\section{Computational Details}

The solvation of 25 small molecules, such as $\mathrm{H}_{2}, \mathrm{~N}_{2}, \mathrm{CO}_{2}$, hydrocarbons (methane, ethane, propane etc.), and haloalkanes $\left(\mathrm{CH}_{3} \mathrm{~F}, \mathrm{CH}_{3} \mathrm{Cl}\right.$, etc.), with water was examined. Geometry optimizations were carried out at the second order Møller-Plesset perturbation (MP2) level with an aug-cc-pVTZ basis set.The electronic energies were evaluated at the $\operatorname{CSSD}(\mathrm{T})$ level with an aug-cc-pVTZ basis set.

The electronic energy in solution was evaluated by the SCRF method, where the conductor-like polarizable continuum model (CPCM) was used. In the CPCM calculations, Bondi's van der Waals radii scaled by $\alpha=1.2$ were used throughout.The repulsion and dispersion terms were evaluated by the method of Floris and Tomasi[40,41].The cavitation energy equation defined by Pierotti includes both enthalpy and entropy contributions in the IGM [42]. The entropy contribution mainly arises from the translational entropy difference between the vapor- and liquid-phases. When the whole cavitation energy is included in the solvation Gibbs energy, the translation entropy component is counted twice in the solvation entropy. This is problematic in the HSM calculations, since the vaporand liquid-phase entropy difference is explicitly taken into account by Eq.(9). For this reason, we only added the enthalpy contribution of the cavitation energy to the solvation enthalpy in the HSM calculations.

Thermodynamic contributions were calculated by the IGM and HSM. All vapor-phase calculations were carried out using Gaussian09, and PCM calculations were carried out using Gaussian09 and GAMESS2013[43,44].

HSM calculations were carried out by our own program connected with the GAMESS software.

Experimental solvation data were taken from the following sources:(i) the IUPAC Solubility Data Series[45], (ii) a review by Wilhelm, Battino, and Wilcock[46], and (iii)data compiled by Sander in which the HLC and 
$\mathrm{d} \ln H_{i} / \mathrm{d}(1 / T)$ were reported with original references[47]. Values of $\mathrm{d} \ln H / \mathrm{d}(1 / T)$ were used for the

estimation of $\Delta H_{\text {solv }}$ from the relationship

$$
\frac{\mathrm{d} \ln H_{i}}{\mathrm{~d}(1 / T)}=\frac{-\Delta H_{\text {solv }}}{R}
$$

When the $\mathrm{HLC}$ is given in $\mathrm{kPa} \cdot \mathrm{m}^{3} \cdot \mathrm{mol}^{-1}$, it is converted to other frequently used units as follows,

$$
\begin{aligned}
& H_{i}^{\prime}\left[\mathrm{atm} \cdot \mathrm{L} \cdot \mathrm{mol}^{-1}\right]=1.0 \times 10^{1} H_{i}\left[\mathrm{kPa} \cdot \mathrm{m}^{3} \cdot \mathrm{mol}^{-1}\right] \\
& H_{i}^{\prime \prime}[\mathrm{GPa}]=1.0 \times 10^{-6} \rho\left[\mathrm{kg} \cdot \mathrm{m}^{-3}\right] / M W\left[\mathrm{~kg} \cdot \mathrm{mol}^{-1}\right] \cdot H_{i}\left[\mathrm{kPa} \cdot \mathrm{m}^{3} \cdot \mathrm{mol}^{-1}\right],
\end{aligned}
$$

where $\rho$ and $M W$ are the density and molecular weight of the solvent, respectively. Solvent density is taken from Ref.[48], where density is fixed to that at $20^{\circ} \mathrm{C}$. 


\section{Results and Discussion}

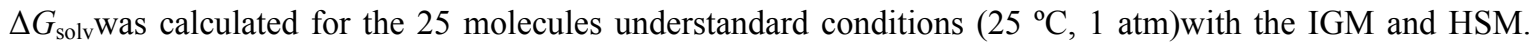

Enthalpy and entropy contributions, i.e. $\Delta H_{\text {solv }}$ and $\Delta S_{\text {solv }}$, were also evaluated. The thermodynamic properties and $\ln H_{i}$ values calculated from $\Delta G_{\text {solv }}$ are shown in Table 1.

As seen from Table 1, the IGM shows reasonable agreement with experimental $\Delta G_{\text {solv }}$ (mean absolute deviation (MAD) of $4.54 \mathrm{~kJ} / \mathrm{mol}$ ). The largest deviation from experimental values wasobtained forCF $4,-10.33$ $\mathrm{kJ} / \mathrm{mol}$. Note that the accuracy ofthe IGMcalculationsis similar to those in previous studies employing the PCM;

Takano and Houk reported an MAD of $5.64 \mathrm{~kJ} / \mathrm{mol}$ for $\Delta G_{\text {solv }}$ calculated by the PCM for neutral species[49].

$\Delta G_{\text {solv }}$ calculated by the HSM shows similar accuracy to that obtained with theIGM, but slightly larger deviation;the

MAD and largest deviation werecalculated as 7.02 and $-22.28 \mathrm{~kJ} / \mathrm{mol}$ (the latter for $\mathrm{CF}_{2} \mathrm{Cl}_{2}$ ), respectively. As a result, $\ln H_{i}$ is of similar accuracy for the IGM and HSM since with MADs of 1.80 and 2.83, respectively.The IGM and HSM therefore show similar accuracy for $\Delta G_{\text {solv }}$ and HLC for neutral gaseous molecules.

Conversely, the IGM and HSM show significantly different results for $\Delta H_{\text {solv }}$ and $\Delta S_{\text {solv }}$. The IGM shows large deviation from experimental values for $\Delta H_{\text {solv }}(\mathrm{MAD}$ of $22.66 \mathrm{~kJ} / \mathrm{mol})$. Likewise, $T \Delta S_{\text {solv }}$ showssignificant errors (MAD of $26.04 \mathrm{~kJ} / \mathrm{mol}$ ).However, the HSM shows good accuracy for both $\Delta H_{\text {solv }}$ and $\Delta S_{\text {solv }}$ (MADs of 6.33 and $6.02 \mathrm{~kJ} / \mathrm{mol}$, respectively).Thus, the accuracy of the entropy component was improved more than 4-foldwith the HSM. Because the conventional PCM scheme only concentrates on the accuracy of $\Delta G_{\text {solv }}$, parameters such as cavity radius were optimized to reproduce experimental $\Delta G_{\text {solv }}$. Accurate individual $\Delta H_{\text {solv }}$ and $\Delta S_{\text {solv }}$ components are then not guaranteed,as clearly shown by the above results. The HSM improves the accuracy of $\Delta S_{\text {solv }}$ more 
significantly than the IGM, and the overall accuracy of $\Delta G_{\text {solv }}$ is similar to or moderately lower than that of the IGM. $\Delta H_{\text {solv }}$ also shows reasonable agreement with experimental values, better than $\sim 10 \mathrm{~kJ} / \mathrm{mol}$ on average. These results show that correct decomposition of $\Delta G_{\text {solv }}$ into $\Delta H_{\text {solv }}$ and $\Delta S_{\text {solv }}$ is possible with the HSM.

The correlation between experimental and theoretical $\Delta H_{\text {solv }}, \Delta S_{\text {solv }}$, and $\Delta G_{\text {solv }}$ values obtained by the IGM and HSM are plotted in Figure 1.It can be seen that theHSM improves the accuracy of both $\Delta H_{\text {solv }}$ and $\Delta S_{\text {solv }}$ significantly. The IGM underestimates $\Delta S_{\text {solv }}$ considerably, since idealgas behavior is assumed in the both vaporand liquid-phases.These results indicate that the IGM performs well for $\Delta G_{\text {solv }}$, but its enthalpy and entropy contributionsare not as accurate.

To analyze the solvation process in more detail, $\Delta H_{\text {solv }}$ and $T \Delta S_{\text {solv }}$ contribution to $\Delta G_{\text {solv }}$ with the IGM and HSM was evaluated, as shown in Figure 2.Five molecules from hydrocarbons, haloalkanes, and small inorganic molecules are shown as examples. The HSM results indicate that rather small $\Delta G_{\text {solv }}$ results from the summation of a large negative $\Delta H_{\text {solv }}$ contribution and large positive $T \Delta S_{\text {solv }}$ contribution. This is in good agreement with experimental understanding, i.e. $\Delta H_{\text {solv }}$ induces the solvation of gaseous molecules via solute-solvent interactions while $T \Delta S_{\text {solv }}$ inhibits solvation since releasing solute molecules into the vapor-phase is entropically favorable. Enthalpic and entropic contributions are of similar magnitude, although $T \Delta S_{\text {solv }}$ contributions slightly exceed those of $\Delta H_{\text {solv }}$ in non-polar solutes such as $\mathrm{CH}_{4}$ and $\mathrm{PH}_{3}$ as a result of smaller solute-solvent interactions. This also correlates with experimental understanding of the solvation of small non-polar molecules, i.e. these processes areentropy-driven[35].As shown in these examples, the correct decomposition of $\Delta G_{\text {solv }}$ into enthalpy and entropy contributions is useful for better understanding of the solvation process, since these components have completely 
different physical origins; larger $\Delta H_{\text {solv }}$ signifies stronger solute-solvent interactions, while larger $\Delta S_{\text {solv }}$ indicates more significant loss of freedom in the liquid-compared to thevapor-phase. This result also indicates that the reasonable accuracy of $\Delta G_{\text {solv }}$ with the IGM is achieved because of the error cancellation of $\Delta H_{\text {solv }}$ and $\Delta S_{\text {solv }}$, where $\Delta H_{\text {solv }}$ and $\Delta S_{\text {solv }}$ are significantly overestimated and underestimated, respectively.

Finally, the temperature dependence of the HLC was investigated.In Figure 3, $\ln H_{i}$ calculated with the IGM and HSMvs.temperature Tis plotted, together with the experimental values. Here, four sets of molecules were employed, (A) alkanes; $\mathrm{CH}_{4}, \mathrm{C}_{2} \mathrm{H}_{6}, \mathrm{C}_{3} \mathrm{H}_{8}$, and $\mathrm{C}_{4} \mathrm{H}_{10}$, (B) haloalkanes; $\mathrm{CH}_{3} \mathrm{~F}, \mathrm{CH}_{3} \mathrm{Cl}$, and $\mathrm{CH}_{3} \mathrm{Br}$, (C) $\mathrm{C}_{2}$-hydrocarbons; $\mathrm{C}_{2} \mathrm{H}_{6}, \mathrm{C}_{2} \mathrm{H}_{4}$, and $\mathrm{C}_{2} \mathrm{H}_{2}$, and (D) small inorganic molecules; $\mathrm{H}_{2}, \mathrm{~N}_{2}, \mathrm{CO}$, and $\mathrm{N}_{2} \mathrm{O}$.To observe the temperature dependence more clearly, relative $\ln H_{i}$ values at $288 \mathrm{~K}$ (the lowest temperature at which experimental data are available) were taken.

As shown in Figure 3, $\ln H_{i}$ increases in the order $\mathrm{C}_{4} \mathrm{H}_{10}, \mathrm{C}_{3} \mathrm{H}_{8}, \mathrm{C}_{2} \mathrm{H}_{6}, \mathrm{CH}_{4}$ for hydrocarbons. The HSM results correctly reproduce this trend, although the increase $\operatorname{in} \ln H_{i}$ is slightly underestimated. On the contrary, the IGM predicts a decrease in $\ln H_{i}$ with increasing temperature. Forhaloalkanes, both the IGM and HSM showincreasingln $H_{i}$ with temperature, although the increase in $\ln H_{i}$ is significantly underestimated with the IGM. Again, the HSM shows reasonable agreement with experimental results in all cases. For small inorganic molecules, the HSM successfully reproduces the experimental order of increasing HLC with temperature $\left(\mathrm{N}_{2} \mathrm{O}>\mathrm{CO}>\mathrm{N}_{2}>\right.$ $\mathrm{H}_{2}$ ), although this was not the case for $\mathrm{C}_{2}$-hydrocabons (correct order: $\mathrm{C}_{2} \mathrm{H}_{6}>\mathrm{C}_{2} \mathrm{H}_{4}>\mathrm{C}_{2} \mathrm{H}_{2}$ ). This inaccuracy results from insufficient treatment of short-range solute-solvent interactions, such as explicit coordination of solvent molecules or intermolecular hydrogen bond formation. Description of these interactions is possible by treating 
several explicit solvent molecules quantum chemically, known as the cluster-continuum approach[50].

The HSM results indicate that the HLC increases with temperature, meaning gaseous solubility decreases at higher temperature since the solubility is inversely proportional to the HLC. This is inaccordance with experimental results, but the IGM fails to reproduce it.This is becausethe IGM does not givethe correct $\Delta S_{\text {solv }}$ since the temperature dependence of $\Delta G_{\text {solv }}$ and the HLC is mainly governed by the entropy component. 


\section{Conclusions}

In the present study, the HSM, which aims to produce accurate thermodynamic properties ofcondensed-phase molecules, was applied to calculation of the HLC, which is equivalent to the solubility of gaseous molecules. The solvation of 25 molecules with water was examined using both the HSM and IGM.

The IGM and HSM show good agreement with experimental values for $\Delta G_{\text {solv }}$. The MADs for $\Delta G_{\text {solv }}$ are 5.60 and $7.02 \mathrm{~kJ} / \mathrm{mol}$, respectively. On the contrary, the IGM fails to reproduce experimental values for $\Delta H_{\text {solv }}$ and $\Delta S_{\text {solv }}$, but the HSM shows considerable improvement. The MADs for $\left\{\Delta H_{\text {solv }}, T \Delta S_{\text {solv }}\right\}$ are $\{21.98,6.33\}$ and $\{90.47$, 20.18 \} in $\mathrm{kJ} / \mathrm{mol}$ for the IGM and HSM, respectively.For most of the examined compounds, $\Delta S_{\text {solv }}$ contribution to $\Delta G_{\text {solv }}$ is larger than that of $\Delta H_{\text {solv }}$ with the HSM, and vice versa for the IGM. The HSM results indicate that the solvation of gaseous molecules is entropy-driven, which agrees well with experimental understanding.

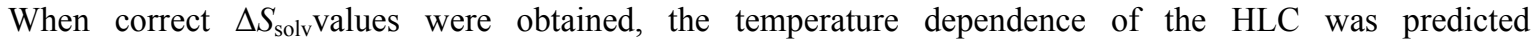
accurately.Experimentally, the HLC decreases as temperature increases. This tendency was correctly reproduced by the HSM but not the IGM. 


\section{Acknowledgements}

Some calculations were performed at the Research Center for Computational Science (RCCS), the Okazaki

Research Facilities, and the National Institutes of Natural Sciences (NINS). This study was supported in part by the

Core Research for Evolutional Science and Technology (CREST) program from the Japan Science and Technology

(JST) Agency, the Grants-in-Aid for Challenging and Exploratory Research "KAKENHI 15K13629”, the Strategic

Programs for Innovative Research (SPIRE), and the Computational Materials Science Initiative (CMSI) from the Ministry of Education, Culture, Sports, Science and Technology (MEXT), Japan. 


\section{References}

[1] J.J. Carroll, J. Chem. Educ. 70 (1993) 91.

[2] D. Mackay, W.Y. Shiu, J. Phys. Chem. Ref. Data 10 (1981) 1175.

[3] J. Altschuh, R. Bruggemann, H. Santl, G. Eichinger, et al., Chemosphere 39 (1999) 1871.

[4] J. Hine, P.K. Mookerjee, J. Org. Chem. 40 (1975) 292.

[5] C.J. Russell, S.L. Dixon, P.C. Jurs, Anal. Chem. 64 (1992) 1350.

[6] A.R. Katritzky, Y.L. Wang, S. Sild, T. Tamm, et al., J. Chem. Inf. Comput.Sci. 38 (1998) 720.

[7] K.U. Goss, Fluid Phase Equilib. 233 (2005) 19.

[8] R.J. Sadus, J. Phys. Chem. B 101 (1997) 3834.

[9] A.Z. Panagiotopoulos, J. Phys.: Condens. Matter.12 (2000) R25.

[10] I.G. Economou, Fluid Phase Equilib. 183 (2001) 259.

[11] J.M. Stubbs, B. Chen, J.J. Potoff, J.I. Siepmann, Fluid Phase Equilib. 183 (2001) 301.

[12] T.I. Morrow, E.J. Maginn, Fluid Phase Equilib. 217 (2004) 97.

[13] J. Vrabec, J. Stoll, H. Hasse, Mol. Simul.31 (2005) 215.

[14] D. Frenkel, B. Smit, Understanding Molecular Simulations, Academic Press, 2002.

[15] M. Lísal, W.R. Smith, K. Aim, Fluid Phase Equilib. 226 (2004) 161.

[16] C.J. Wu, X.F. Li, J.X. Dai, H. Sun, Fluid Phase Equilib. 236 (2005) 66.

[17] H. Yuan, S. Murad, C.J. Jameson, J.D. Olson, J. Phys. Chem. C. 111 (2007) 15771.

[18] G.C. Boulougouris, J.R. Errington, I.G. Economou, A.Z. Panagiotopoulos, et al., J. Phys. Chem. B 104 (2000) 4958.

[19] T. Schnabel, J. Vrabec, H. Hasse, J. Mol. Liq. 135 (2007) 170.

[20] A.Z. Panagiotopoulos, N. Quirke, M. Stapleton, D.J. Tildesley, Mol. Phys. 63 (1988) 527.

[21] W.R. Smith, B. Triska, J. Chem. Phys. 100 (1994) 3019.

[22] M. Lísal, W.R. Smith, I. Nezbeda, J. Phys. Chem. B 103 (1999) 10496.

[23] J. Tomasi, M. Persico, Chem. Rev. 94 (1994) 2027.

[24] J. Tomasi, B. Mennucci, R. Cammi, Chem. Rev. 105 (2005) 2999. 
[25] G. Schüürmann, J. Comput. Chem. 21 (2000) 17.

[26] E.J. Delgado, J.B. Alderete, J. Chem. Inf. Comput. Sci. 42 (2002) 559.

[27] E.J. Delgado, J.B. Alderete, J. Chem. Inf. Comput. Sci. 43 (2003) 1226.

[28] X. Zhang, Y. Zeng, Fluid Phase Equilib. 376 (2014) 234.

[29] Y. Zeng, X. Chen, D. Zhao, H. Li et al., Fluid Phase Equilib.313 (2012) 148.

[30] A. Klamt, F. Eckert, Fluid Phase Equilib. 172 (2000) 43.

[31] A. Klamt, F. Eckert, Fluid Phase Equilib. 217 (2004) 53.

[32] S. Oleszek-Kudlak, M. Grabda, E. Shibata, F. Eckert, et al., Environ. Toxicol.Chem. 24 (2005) 1368.

[33] B. Schroeder, L.M.N.B.F. Santos, I.M. Marrucho, J.A.P. Coutinho, Fluid Phase Equilib. 289 (2010) 140.

[34] H. Nakai, A. Ishikawa, J. Chem. Phys. 141 (2014) 174106.

[35] J.H. Hildebrand, R.L. Scott, The solubility of nonelectrolytes, Reinhold Publishing, New York, 1950.

[36] E.J. Baum, Chemical Property Estimation; Theory and Application, CRC Press, 1997.

[37] D. Kondepudi, Introduction to Modern Thermodynamics, John Wiley \& Sons, 2007.

[38] J.M. Smith, H.C. Van Ness, M.M. Abbott, Introduction to Chemical Engineering Thermodynamics, McGraw-Hill, New York, 2001.

[39] A. Ben-Naim, Solvation Thermodynamics, Plenum Press, New York, 1987.

[40] F.M. Floris, J. Tomasi, J. Comput. Chem. 10 (1989) 616.

[41] F.M. Floris, J. Tomasi, J.L.P. Ahuir, J. Comput. Chem. 12 (1991) 784.

[42] R.A. Pierotti, Chem. Rev. 76 (1976) 717.

[43] M.J. Frisch, G.W. Trucks, H.B. Schlegel, G.E. Scuseria, et al., Gaussian 09, Revision C.01. Gaussian Inc, Wallingford, CT, 2009.

[44] M.W. Schmidt, K.K. Baldridge, J.A. Boatz, S.T. Elbert, et al., J. Comput.Chem. 14 (1993) 1347.

[45] IUPAC, Solubility Data Series, Pergamon, 1972.

[46] E. Wilhelm, R. Battino, R.J. Wilcock, Chem. Rev. 77 (1977) 219.

[47] R. Sander, Atmos. Chem. Phys. Discuss.14 (2014) 29615.

[48] CRC Handbook of Chemistry and Physics, CRC Press, Boca Raton, FL., 2014. 
[49] Y. Takano, K.N. Houk, J. Chem. Theory Comput.1 (2005) 70.

[50] D.G. Truhlar, J.R. Pliego, in: B. Mennucci, R. Cammi (Eds.), Continuum Solvation Models in Chemical Physics, John Wiley \& Sons Ltd, England, 2007, p. 338. 


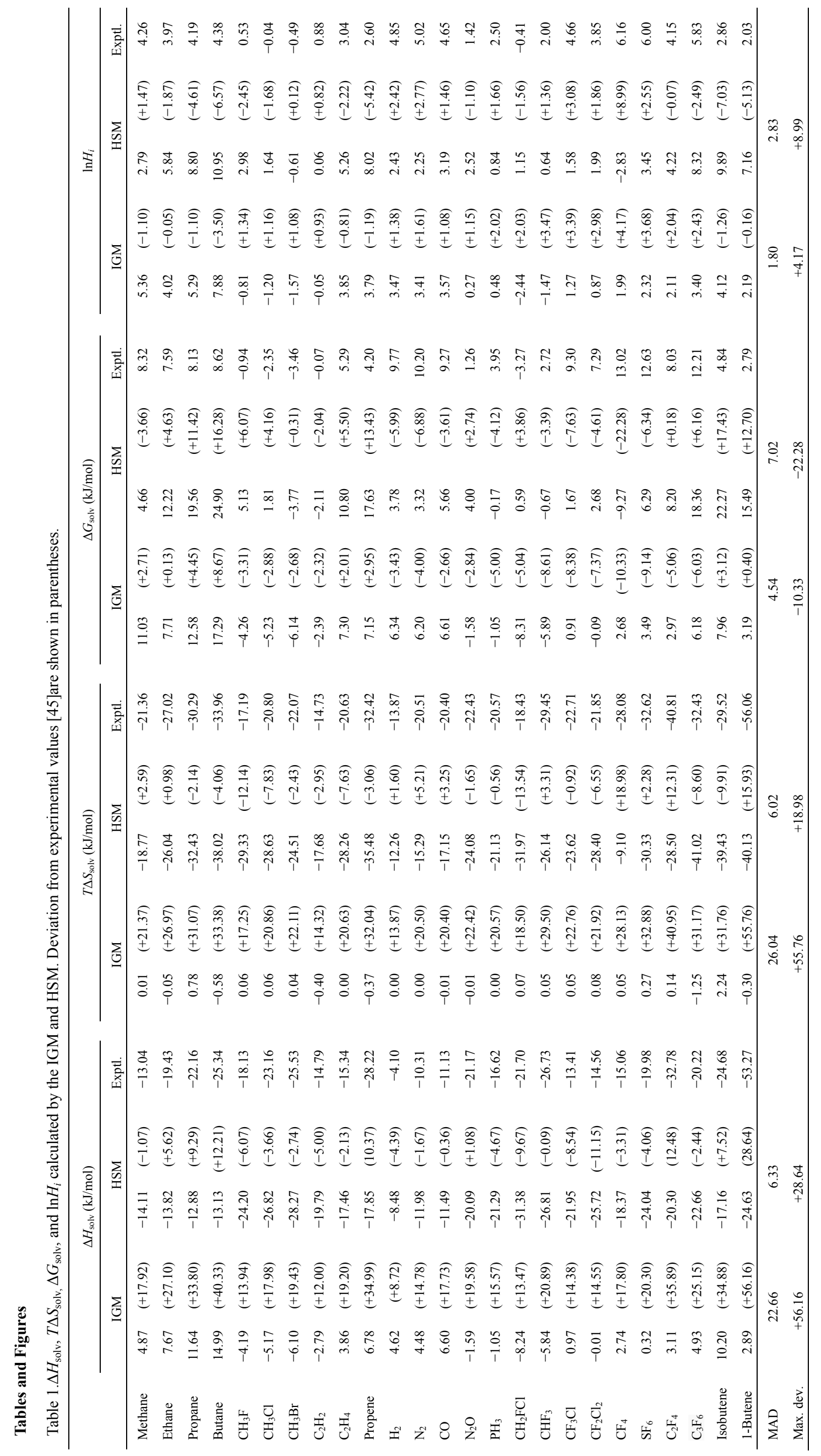




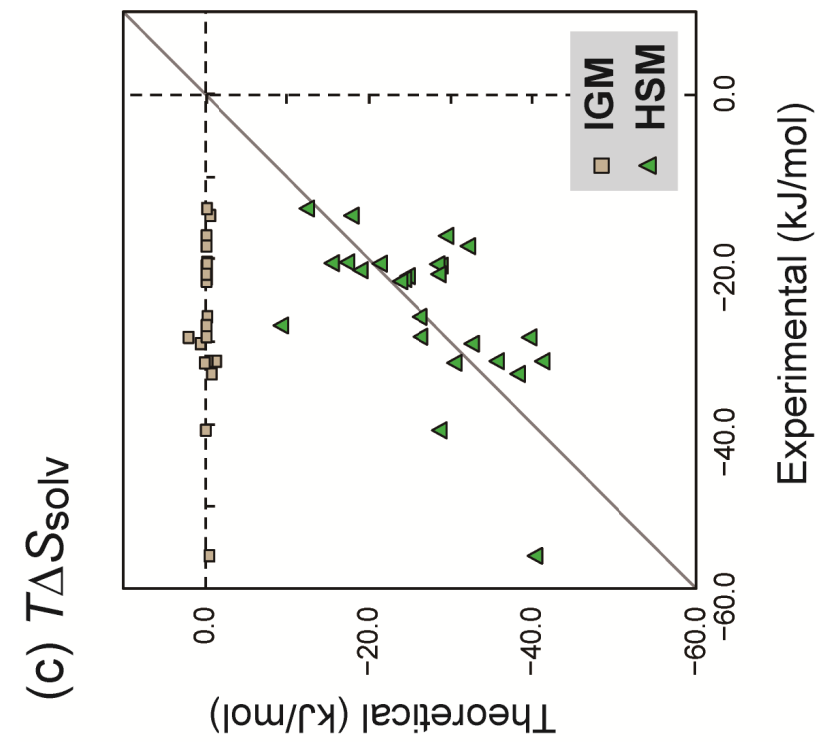


(A) Hydrocarbons
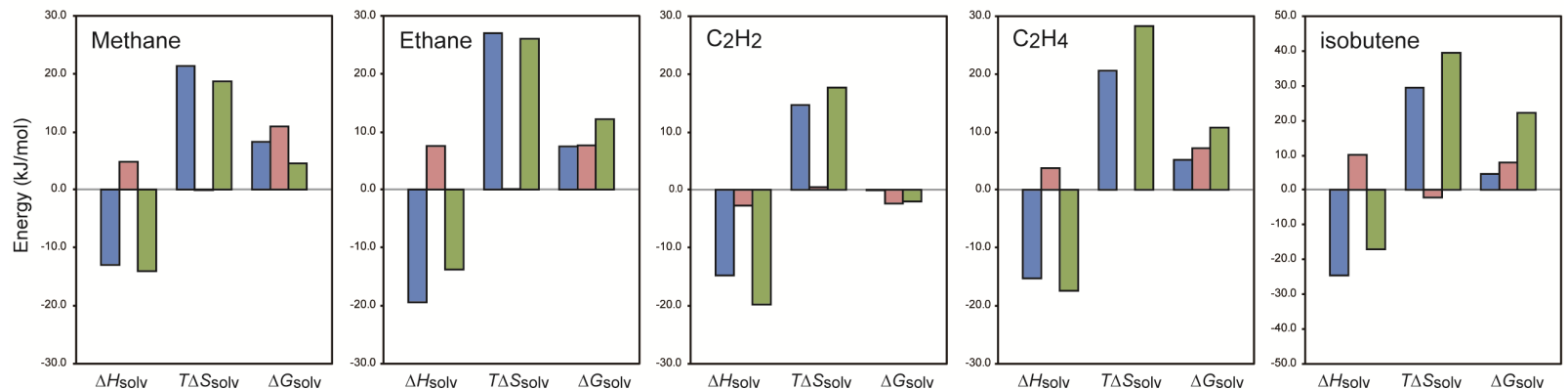

(B) Haloalkanes
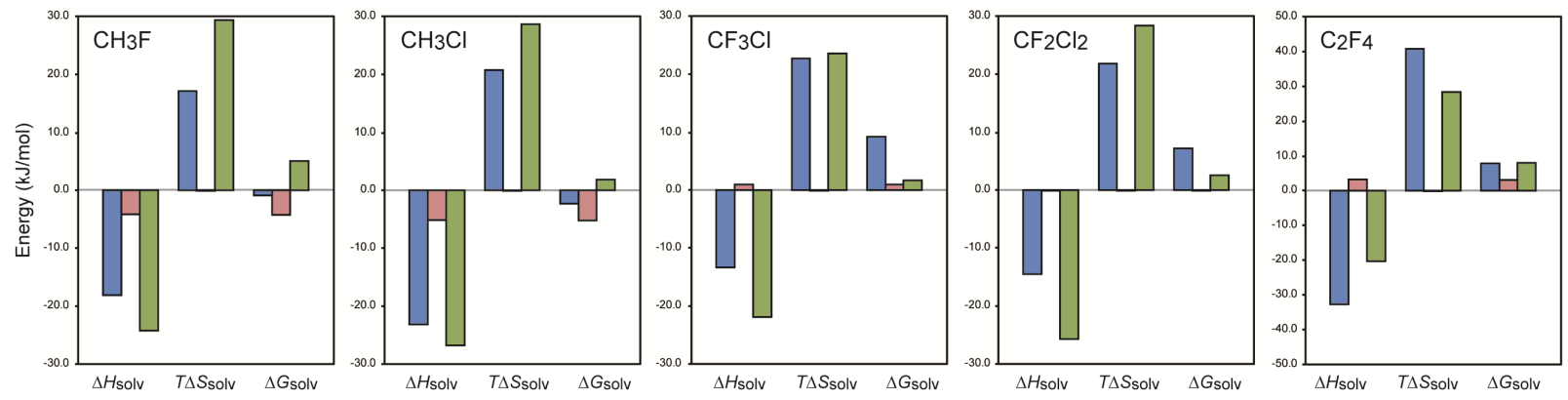

(C) Small inorganic molecules
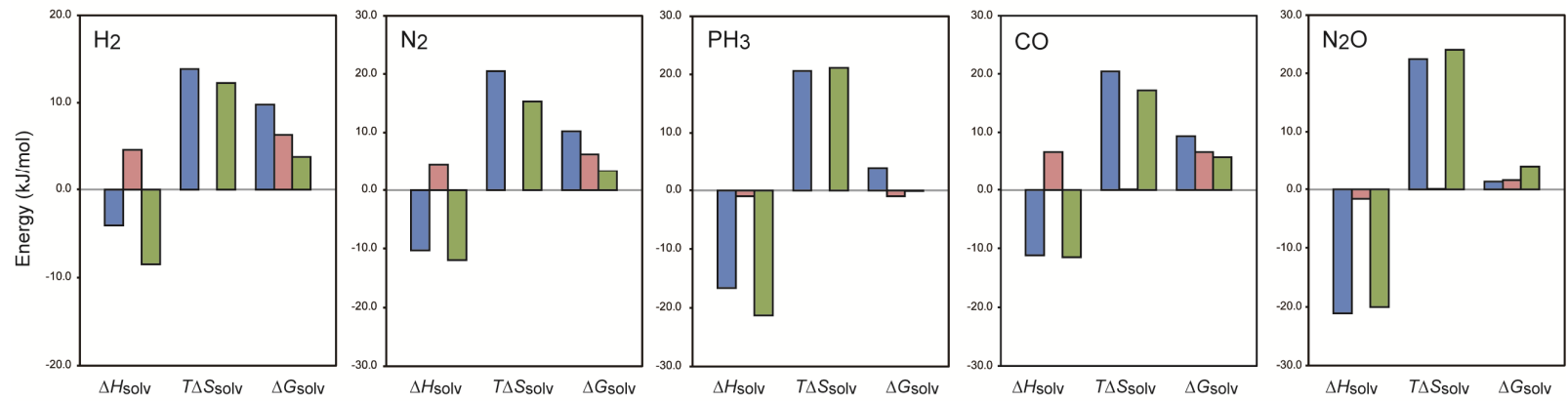

Figure 2.Contribution of $\Delta H_{\text {solv }}$ and $T \Delta S_{\text {solv }}$ to $\Delta G_{\text {solv }}$ for (A) hydrocarbons, (B) haloalkanes, and (C) small inorganic molecules. Under standard conditions (298.15 K, 1 atm). Experimental values (Ref.[45]) and IGM and HSM computational results are shown. 
(a)

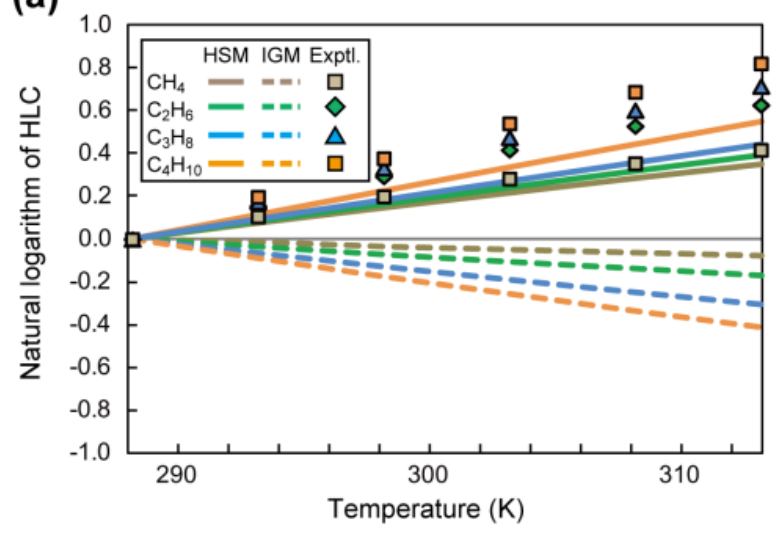

(c)

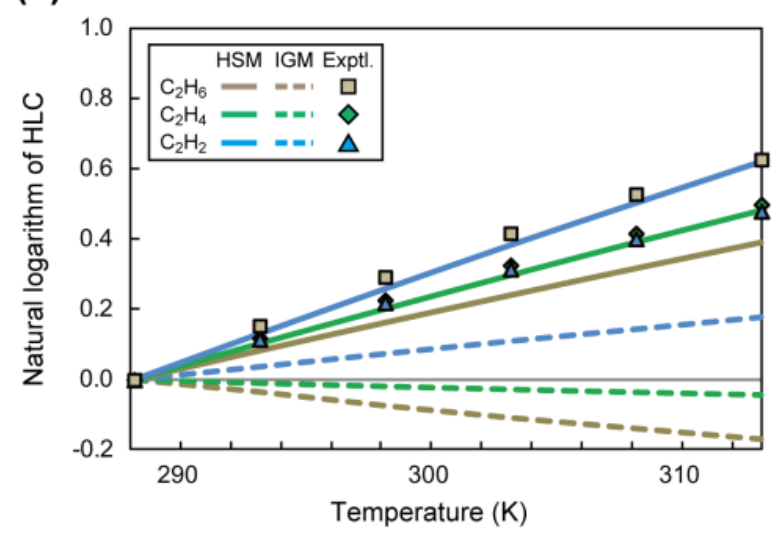

(b)

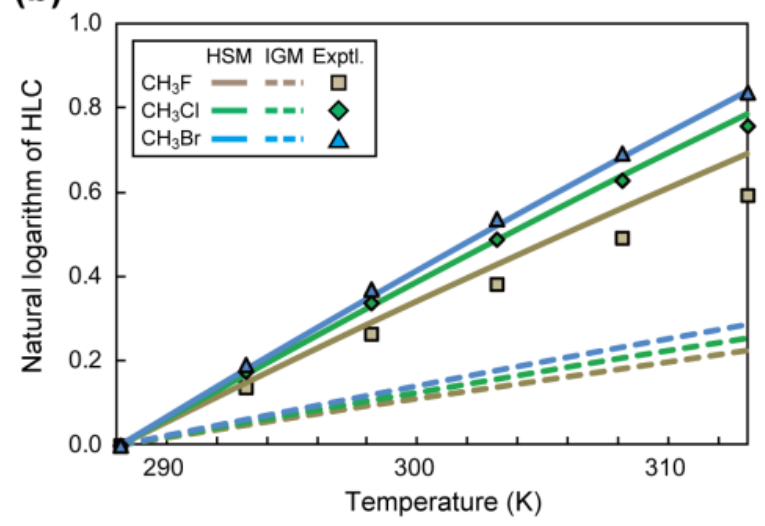

(d)

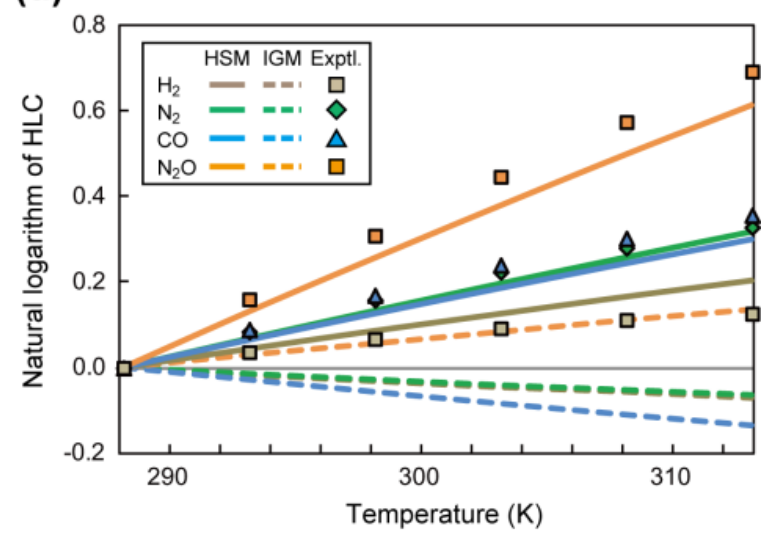

Figure 3.Relative temperature dependence of HLC for (A) alkanes, (B) haloalkanes, (C) $\mathrm{C}_{2}$-hydrocarbons, and (D) small inorganic molecules. Experimental values were taken from Ref.[47], and theoretical values were obtained using the IGM and HSM. 


\section{Graphical Abstract}

\section{Enthalpic and Entropic Contributions to Gaseous Solubility}

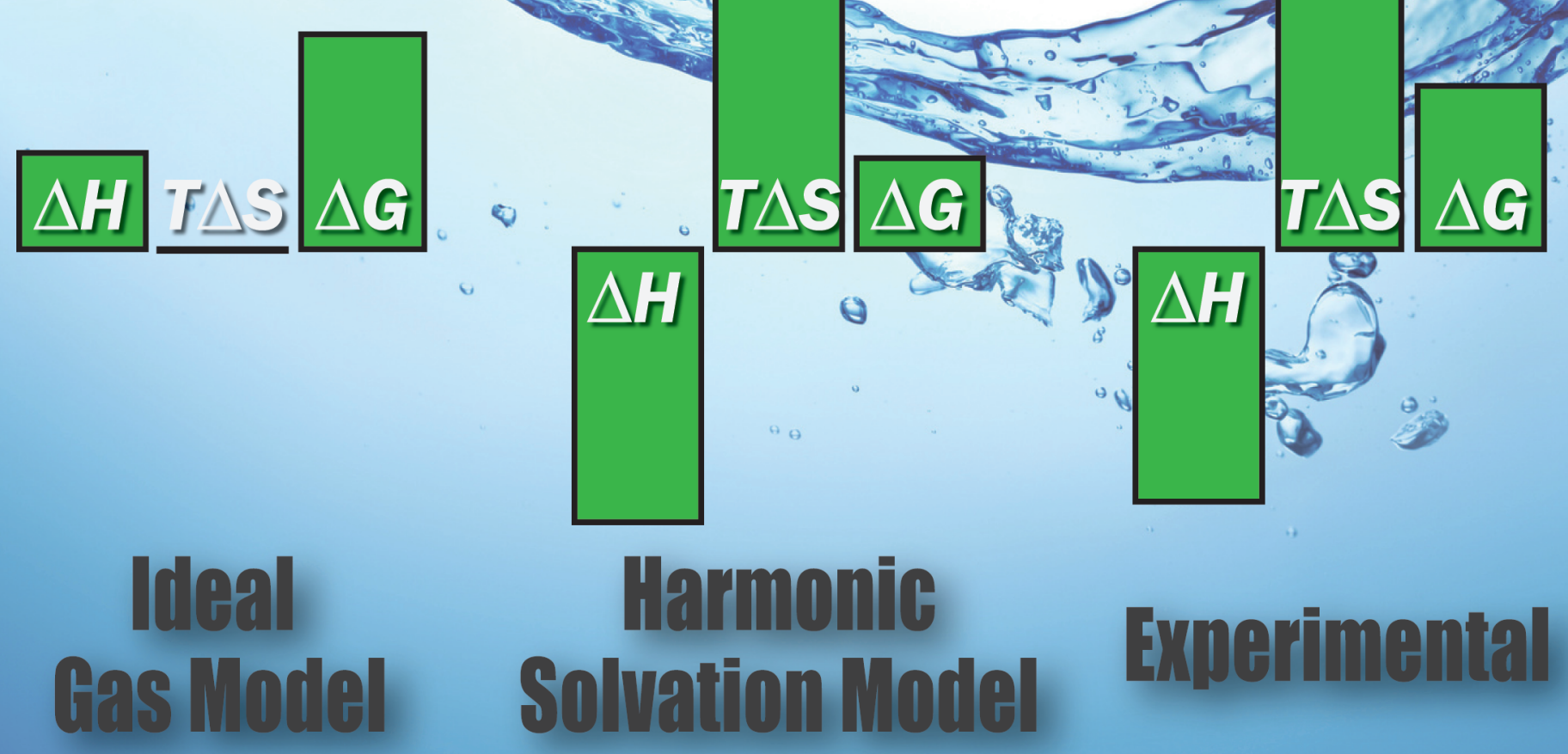

